\title{
Repository Info
}

National Cancer Institute

\section{Source}

National Cancer Institute. Repository Info. NCI Thesaurus. Code C41119.

An object that contains information about the availability of a particular model from the repository. A submitter of data to the cancer models database can also indicate if their model should be submitted to the repository. 